\title{
Preliminary study on the inhibitory effect of tumor suppressor gene KPC1 on the proliferation in gastric carcinoma cell
}

\author{
Min Zhou ${ }^{1 \#}$, Chun-Ying Qu ${ }^{1 \#}$, Wen-Jin Ding ${ }^{2}$, Xiao-Lei Cai ${ }^{1}$, Yu-Jie Shen ${ }^{1}$, Ying-Wei Chen ${ }^{2}$, \\ Lei-Ming $\mathrm{Xu}^{1}$
}

${ }^{1}$ Digestive Endoscopic Diagnosis and Treatment Center, ${ }^{2}$ Department of Gastroenterology, Xin Hua Hospital Affiliated to Shanghai Jiao Tong University School of Medicine, Shanghai 200092, China

Contributions: (I) Conception and design: LM Xu, YW Chen; (II) Administrative support: LM Xu, YW Chen; (III) Provision of study materials or patients: WJ Ding, X Cai, YJ Sun; (IV) Collection and assembly of data: CY Qu; (V) Data analysis and interpretation: M Zhou, CY Qu; (VI) Manuscript writing: All authors; (VII) Final approval of manuscript: All authors.

"These authors contributed equally to this work.

Correspondence to: Dr. Lei-Ming Xu. Chief Physician, Digestive Endoscopic Diagnosis and Treatment Center, Xin Hua Hospital Affiliated to Shanghai Jiao Tong University School of Medicine, No. 1665, Kongjiang Road, Yangpu District, Shanghai 200092, China. Email: xuleiming@xinhuamed.com.cn; Dr. Ying-Wei Chen. Chief Physician, Department of Gastroenterology, Xin Hua Hospital Affiliated to Shanghai Jiao Tong University School of Medicine, Shanghai 200092, China. Email: chenyingwei@xinhuamed.com.cn.

Background: To investigate Kip1 ubiquitination-promoting complex 1 (KPC1) expression and its relationship with $\mathrm{NF}-\kappa \mathrm{B}$ p50 in gastric cancer cell lines.

Methods: The expression of KPC1 and NF- $\mathrm{BB}$ p50 in tissue samples from 159 gastric cancer patients after tumor resection and normal gastric mucosa samples from 56 patients as negative controls was retrospectively studied. The relationship between KPC1, NF- $\kappa \mathrm{B}$ p50, and clinicopathological factors was analyzed, and the correlation between KPC1 and cytoplasmic NF- $\mathrm{BB}$ p50 was determined. The expression level of KPC1 and NF- $\mathrm{B}$ p50 was researched using reverse transcription (RT) polymerase chain reaction (RT-PCR) and Western blotting in 3 differentiated human gastric cancer cell lines (AGS, SGC-7901 and MGC-803).

Results: Immunohistochemistry indicated that KPC1 and NF- $\kappa$ B p50 expression was significantly decreased in gastric cancer cases, and the level of expression varied across the differentiated gastric cancer tissues. KPC1 and NF- $\mathrm{BB}$ p50 expression was significantly connected with tumor differentiation, tumornode-metastasis (TNM) staging, and metastasis of 159 patients suffering from gastric cancer $(\mathrm{P}<0.05)$, but not correlated with age and lesion size $(\mathrm{P}>0.05)$. KPC1 was positively connected with the expression of $\mathrm{NF}-$ $\kappa \mathrm{B}$ p50 by the Spearman correlation analysis $(\mathrm{r}=0.427, \mathrm{P}<0.05)$. The expression of KPC1 and NF- $\mathrm{kB}$ p50 mRNA was reduced, and there were differences in the 3 differentiated human gastric cancer cell lines, as confirmed by western blotting.

Conclusions: The co-expression of KPC1 and cytoplasmic NF- $\mathrm{KB}$ p50 in gastric cancer promotes tumor suppressor gene expression. Therefore, limiting the growth of tumor cells may inhibit the development of gastric cancer.

Keywords: Gastric cancer; tumor suppressor gene; Kip1 ubiquitination-promoting complex 1 (KPC1); ubiquitination; target

Submitted Dec 24, 2019. Accepted for publication Feb 04, 2020.

doi: 10.21037/atm.2020.02.79

View this article at: http://dx.doi.org/10.21037/atm.2020.02.79 


\section{Introduction}

Gastric cancer has one of the highest incidences among malignancies in China. The morbidity and mortality related to gastric cancer are high not only in China but also across the world, and gastric cancer is a major cause of global cancer-related deaths (1-4). However, gastric cancer genetic and epigenetic mechanisms have not been fully elucidated, resulting in difficulties in its prevention and treatment. While the emergence and development of gastric cancer is known to be closely related to the inactivation of multiple tumor suppressor genes and/or oncogene activation, the relationship between the tumor suppressor genes and gastric cancer is not sufficiently understood. Therefore, attention should be paid to the function of tumor suppressor genes in the development and progression of gastric cancer, particularly those clinicopathological features and specific molecular mechanisms related to the early diagnosis of gastric cancer. A greater understanding of these phenomena may facilitate the design of rational drug treatments, enhance the ability to predict prognosis, and improve diagnosis and treatment.

Ubiquitin is a highly conservative polypeptide consisting of 76 amino acid residues. The pathway of protein degradation mediated by ubiquitin is a specific protein degradation pathway that is strictly and spatiotemporally regulated. Under the catalytic action of adenosine triphosphate (ATP) ubiquitin is transferred to the substrate by ubiquitin ligase enzyme E3, and the polyubiquitinmodified substrate protein finally enters the proteasome for degradation. Subsequently, E3 recruits specific substrates and ubiquitin ligase enzyme E2, and mediates the transfer of process ubiquitin from E2 to the target protein. Therefore, E3 plays a crucial role in the degradation of the entire protein and determines the specificity of the reaction. The ubiquitin protease pathway (UPP) is an important and critical pathway in cell function, and is in charge of the degradation of deficient proteins. If defective proteins are not removed, they may cause cell stress damage. Ubiquitin systems can label these defective proteins and degrade them through the intracellular proteasome pathway. These systems also eliminate functional and healthy proteins that are no longer required by adjusting the processes controlled by those proteins (5). The available data indicate that aberrant activation or inactivation of the UPP is directly related to the occurrence and development of a variety of tumors, including those of gastric cancer (6).

The signaling pathway of $\mathrm{NF}-\mathrm{\kappa B}$ is closely related to cell proliferation, inflammation, survival, and cell changes. The role of NF- $\kappa \mathrm{B}$ p65 is the best known among the $\mathrm{NF}-\kappa \mathrm{B}$ family of proteins. When p65 enters the nucleus, it can promote the transcription of multiple genes, eventually leading to cell growth and the evolution of tumors.

Recent studies have found that Kip1 ubiquitinationpromoting complex 1 (KPC1) has ubiquitin ligase activity, and the ubiquitination of KPC1 can regulate NF-кB1 p65 through the ubiquitin proteasome degradation pathway. After degradation, it may form active p50, which can promote tumor suppressor signal expression, with high expression capable of downregulating p65 and oncogene expression (7). These studies have also found that, compared with normal human tissues, KPC1 and p50 expression in human tumor cells is lower, indicating that KPC1 may contribute to inhibiting tumorigenesis by adjusting p50 expression in the NF- $\mathrm{KB}$ signaling pathway (8-11). Despite this intriguing phenomenon, research on $\mathrm{KPC} 1$ is very limited. The role, mechanism, and clinical characteristics of KPC1 in gastric cancer have not yet been reported. If $\mathrm{KPC} 1$ has a role in suppressing gastric cancer and has clinical significance, it may be used for gastric cancer cases as a prognostic biomarker and may serve as a new target for antitumor drug design.

The purpose of this study was to evaluate the biological function and clinical significance of KPC1 in gastric cancer, and to explore the molecular mechanism of KPC1 inhibition of tumorigenesis. The study may provide new methods and ideas for further research on biopharmaceutical and gastric cancer treatment options.

\section{Methods}

\section{Ethics statement}

The study was approved by the ethics committee of Xin Hua Hospital Affiliated to Shanghai Jiao Tong University School of Medicine, and written informed consent of each patient enrolled during the research was acquired.

\section{Patients}

From September 2015 to October 2017, the clinicopathological data of 159 gastric cancer patients who experienced surgical operation at Xin Hua Hospital affiliated to Shanghai Jiao Tong University School of Medicine, Shanghai, China, were retrospectively analyzed. The average age was $53.1 \pm 10.3$ years old. In addition, normal 
gastric mucosa samples from 56 patients were included as negative controls. Patients who conformed to the following evaluation criteria were included: (I) gastric adenocarcinoma diagnosed by gastroscopy at the Department of Digestive Endoscopic Diagnosis and Treatment and identified by gastric mucosal histopathological examination; (II) past surgical history that included gastrectomy; (III) complete follow-up data available; (IV) no pre-operative therapy, such as chemotherapy or radiotherapy; (V) no history of other synchronous malignancy (such as esophageal cancer, gastrointestinal stromal tumors (GISTs) or colorectal cancer); and (VI) patient diagnosis was made by experienced endoscopic physicians, and tumor resection was performed by experienced surgeons.

Fresh gastric cancer tissue samples were acquired by gastroscopy from patients at the Department of Digestive Endoscopic Diagnosis and Treatment between 2015 and 2017, and the samples were confirmed by histopathological examination. The samples embedded by paraffin were acquired from the 159 gastric cancer patients who underwent surgery at the Xin Hua Hospital between 2015 and 2017. Each tumor sample was graded histologically according to the World Health Organization (WHO) classification standards, and all patients were staged based on the 7th edition of the International Union Against Cancer (UICC) tumor-node-metastasis (TNM) staging system.

\section{Cell line culture and proliferation}

The following 3 human gastric cancer cell lines were acquired from the Laboratory of Digestion, Xin Hua Hospital: AGS, SGC-7901, and MGC-803. Of these, AGS was a well-differentiated gastric adenocarcinoma cell line, SGC-7901 was a moderately differentiated gastric adenocarcinoma cell line, and MGC-803 was a poorly differentiated gastric adenocarcinoma cell line. These cells were cultured by Dulbecco's Modified Eagle Media (DMEM, Gibco/Life technologies Company, USA) culture medium complemented with $10 \%$ fetal calf serum.

Based on their tumorigenicity in nude mice, the cells were divided into strong tumorigenic cell lines (SGC-7901, MGC-803) and weak tumorigenic cell lines (AGS), and subsequently cultured in DMEM (Invitrogen) with 10\% fetal calf serum used as supplement. The expression levels of KCP1 and NF- $\mathrm{KB}$ p50 in these cells were evaluated by reverse transcription (RT) polymerase chain reaction (RT-PCR) and western blotting.
For RNAi experiments, cells were pretreated for $24 \mathrm{~h}$ with $20 \mathrm{nmol} / \mathrm{L}$ KPC1 siRNA or a scrambled control.

SN50, a newly designed peptide comprising 26 amino acids, possesses a lipophilic cell membrane-permeable motif and a nuclear localization sequence which specifically compete with the nuclear localization sequence of the $\mathrm{NF}-\kappa \mathrm{B}$ p50 subunit. Distilled sterilization water was used to dilute SN50 (BIOMOL, Plymouth Meeting, PA, USA) in order to create a stock solution which was stored according to the instructions of manufacturer. Based on the protocol of previous in vitro and in vivo experiments (12), a concentration of $18 \mu \mathrm{mol} / \mathrm{L}$ of the SN50 solution was used as the final concentration.

As instructed by the manufacturer (Roche Diagnostics, Mannheim, Germany), the cell proliferation evaluated by the WST-1 assay was based on tetrazolium salt WST-1 (4-[3-(4-iodophenyl)-2-(4-nitrophenyl)-2H-5-tetrazolio]1,3-benzene disulphonate), and the MGC-803 cell was chosen due to its strong tumorigenicity.

\section{Immunobistochemical analysis}

Dimethylbenzene was used to deparaffinize the tissue sections with rehydration being completed with $100 \%$, $95 \%, 90 \%, 80 \%$, and $70 \%$ ethanol. After being washed 3 times with phosphate-buffered saline (PBS), the slides were heated in antigen retrieval buffer comprising $0.01 \mathrm{M}$ sodium citrate-hydrochloric acid ( $\mathrm{pH}$ 6.0) for about $15 \mathrm{~min}$ in a microwave oven. After a rinsing with PBS, primary antibodies were used for incubating the tissue sections, which was followed by another rinsing of the slides with $3 \%$ peroxidase quenching solution (Invitrogen Company, USA) in order to block endogenous peroxidases. The sections were then incubated using mouse anti-human KPC1 monoclonal antibody (Abcam Company, UK, at a 1:100 dilution) and rabbit anti-human NF- $\mathrm{BB}$ p50 polyclonal antibody (Thermo Fisher Scientific Company, USA) at a 1:500 dilution overnight at $4{ }^{\circ} \mathrm{C}$. This was followed by incubation with secondary antibody for about $30 \mathrm{~min}$ at room temperature. After being washed with PBS once more, the sections then were recruited with 3,3'-diaminobenzidine (DAB) solution, and every slide was counterstained using hematoxylin. The normal gastric mucosa sections (negative controls) were handled as described above, except that they were incubated overnight at $4{ }^{\circ} \mathrm{C}$ in bovine serum albumin (BSA) without the primary antibody.

The expression level of KCP1 and NF- $\kappa \mathrm{B}$ p50 was defined as follows: "-" (score 0 , negative), “+” (score 1, 
weakly positive), “++" (score 2, positive), and “+++" (strongly positive, score 3). A score of 0 and 1 was identified as a low expression, and a score of 2 and 3 was identified as a high expression.

The final KCP1 and NF- $\mathrm{kB}$ p50 immunostaining score was calculated the percentage of positive cells within a total of about 1,500 tumor cells. Briefly, NF-кB p50 0-5\% staining was considered nuclear negative, $>5 \%$ staining was considered positive, and the cytoplasmic staining classification was similar to that of KPC1.

Based on the KCP1 and NF- $\mathrm{KB}$ p50 expression levels, the gastric cancer patients were placed into 2 groups: the low expression group and the high expression group.

\section{Extraction of total RNA and RT-PCR}

Total RNA was extracted with Trizol (Invitrogen, Carlsbad, CA, USA) as per the manufacturer's protocol. The total RNA concentration was assessed by determining the absorbance at $260 \mathrm{~nm}$ with a NANO DROP spectrophotometer (ND-1000, Thermo Fisher Scientific, USA). RT for compounding first-strand cDNA was completed with $10 \mu \mathrm{L}$ of total RNA and water (total RNA was calculated based on the concentration of RNA tested), and dealt with by M-MLV reverse transcriptase (Promega, USA) as per the instructions of the manufacturer. The resulting cDNA was then followed by RT-PCR for assessment of the relative mRNA levels of KCP1, NF- $\kappa \mathrm{B}$ p50, and glyceraldehyde3-phosphate dehydrogenase (GAPDH) (the internal control) with the following primers: KCP1: forward, 5'-GTGGGTGTCTCCGATGATGTC-3', reverse, 5'-CAAGGATGTCCTTCCTCCTCTT-3'; NF-кB р50: forward, 5'-AAGAAGAAGTGCAGAGGAAACG-3', reverse, 5'-CCCACCATAAGTAGGAAATCCA-3'; GAPDH: forward, 5'-CAAGGATGTCCTTCCTCCTCTT-3', and reverse 5'-GCCAGTAGAGGCAGGGATGATGTTC-3. Gene-specific amplification was implemented with a PCR system with $25 \mu \mathrm{L}$ of PCR Mix containing $1 \mu \mathrm{L}$ cDNA, $2 \mu \mathrm{L}$ dNTPs $(10 \mathrm{mM}), 2.5 \mu \mathrm{L} 10 \times$ PCR buffer, $1 \mu \mathrm{L} \mathrm{MgCl}_{2}$, $2 \mu \mathrm{L}$ primers, $0.2 \mu \mathrm{L}$ Taq, and $16.3 \mu \mathrm{L}$ dd $\mathrm{H}_{2} \mathrm{O}$. The mixture was preheated to $95^{\circ} \mathrm{C}(5 \mathrm{~min})$ and then amplified at $95{ }^{\circ} \mathrm{C}(30 \mathrm{~s})$ and $58^{\circ} \mathrm{C}(30 \mathrm{~s})$, and extended at $72{ }^{\circ} \mathrm{C}(30 \mathrm{~s})$ for 32 cycles, and finally extended at $72{ }^{\circ} \mathrm{C}$ for $5 \mathrm{~min}$. The geometric mean of the internal control gene, GAPDH, was used to standardize the relative expression levels of the target genes. All samples were checked repeatedly, and every experiment was repeated at least 2 times with cDNA samples separated from RT reactions.

The product was detected by gel electrophoresis. When electrophoresis was complete, the gel was removed, and the electrophoresis results were observed using the Chimi DoxXRS gel imager scanning system. The molecular weight of the amplified fragment was assessed according to the DNA marker gradient value, and the target band and internal parameters with the gray ratio.

\section{Western blot analysis}

The AGS, SGC-7901, and MGC-803 cancer cell lines were homogenized with RIPA lysis buffer, and the lysates were purified by centrifugation $(12,000 \mathrm{rpm})$ for $15 \mathrm{~min}$ $4{ }^{\circ} \mathrm{C} .40 \mathrm{mg}$ protein samples were run on $10 \%$ SDS-PAGE gels and transferred to PVDF membranes. After nonspecific binding sites were blocked using $5 \%$ non-fat milk for $90 \mathrm{~min}$, the membranes were incubated overnight at $4{ }^{\circ} \mathrm{C}$ with a $1: 1,000$ dilution of both mouse anti-human KPC1 monoclonal antibody (Abcam Company, UK) and

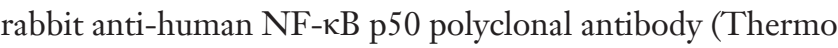
Fisher Scientific Company, USA). The membranes were then washed 3 times using $1 \times$ TBS-T for $10 \mathrm{~min}$ each time and probed with a horseradish peroxidase (HRP)conjugated secondary antibody (Immunology Consultants Laboratory, USA) with a 1:5,000 dilution for $2 \mathrm{~h}$ at room temperature. Finally, the membranes were washed 3 times using $1 \times \mathrm{TBS}-\mathrm{T}$ and added enhanced chemiluminescence system (ECL, Pierce).

\section{Statistical analysis}

SPSS 19.0 statistical software was used for statistical analysis. The experimental data are expressed as the mean \pm standard deviation. All experiments were completed in triplicate. The data from the 2 groups were contrasted using the independent samples $t$-test and the Pearson $\chi^{2}$ test. The significance level was set at $\alpha=0.05$ (bilateral), and $\mathrm{P} \leq 0.05$ was recognized as a statistically significant value. Correlation analysis was performed using Spearman's correlation, and $\mathrm{P}<0.05$ was recognized as a statistically significant value.

\section{Results}

\section{Expression of KPC1 in gastric cancer}

Immunohistochemistry showed that KPC1 was positioned on the cytoplasm, different pathological types of gastric 


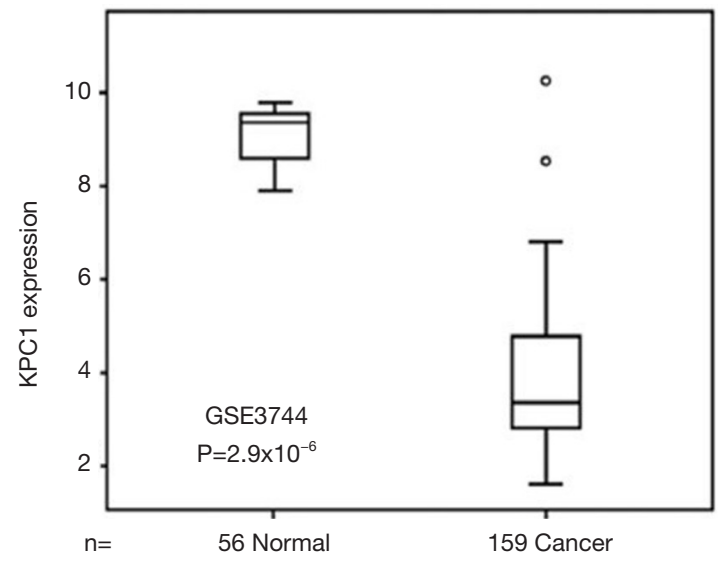

Figure 1 Reduced expression of KPC1 in gastric cancer patients. KPC1, Kip1 ubiquitination-promoting complex 1.

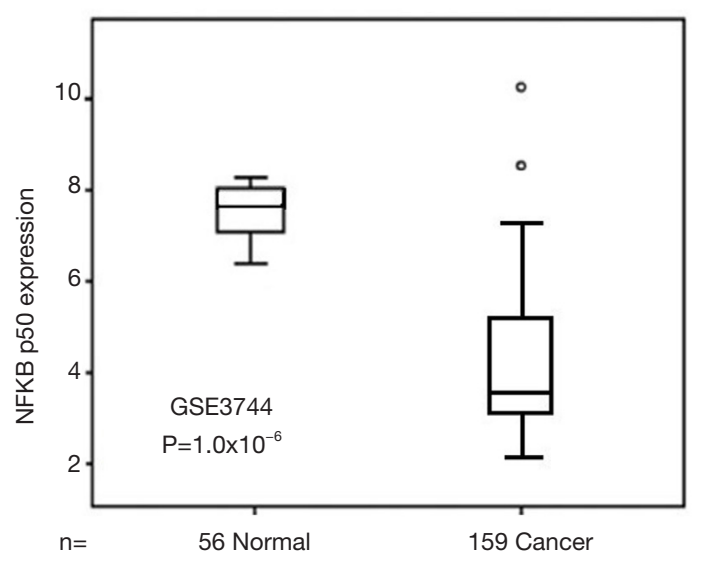

Figure 2 Reduced expression of $\mathrm{NF}-\kappa \mathrm{B}$ p50 in gastric cancer patients.

cancer tissues were visible as brown particles, and the percentage of different, randomly selected stained tissue sections from 159 gastric cancer cases was different from 56 normal gastric tissue. KPC1 expression was also seen in normal gastric tissue, but the expression was different from that in gastric cancer tissue (Figure 1).

\section{Expression of $N F-\kappa B$ p50 in gastric cancer}

Immunohistochemistry showed that NF- $\kappa \mathrm{B}$ p50 was distributed throughout gastric cancer tissue, and was located in the cytoplasm and/or the nucleus. The different pathological types of gastric cancer tissues were visible as brown particles, and the percentage of different, randomly selected stained tissue sections from gastric cancer cases was different from that in normal gastric tissue. Meanwhile, the $\mathrm{NF}-\kappa \mathrm{B}$ p50 expression found in 56 normal gastric tissues was different from that in 159 gastric cancer tissues (Figure 2).

\section{Expression of KPC1 in various differentiated gastric cancer tissues based on immunobistochemical staining}

Immunohistochemistry analysis showed that the distribution density of KPC1-stained brownish yellow particles varied across differentiated tissue types: (I) brownish yellow granules were densely distributed in highly differentiated tissues, and KPC1 expression was considered strongly positive; (II) brown particles were distributed in moderately differentiated tissue, and KPC1 expression was considered moderately positive; (III) brownish yellow particles were scattered in poorly differentiated tissue and KPC1 expression was considered weakly positive (Figure 3).

\section{Expression of NF-кB p50 in various differentiated gastric cancer tissues based on immunobistochemical staining}

Immunohistochemistry showed that NF- $\kappa$ B p50 was scattered in the tissue sections, but localized in the cytoplasm and/or nucleus. The expression level of NF- $\kappa \mathrm{B}$ p50 in well-differentiated gastric cancer tissues was higher in the cytoplasm than in the nucleus. NF- $\kappa \mathrm{B}$ p50 brown particles were found in the nucleus in poorly differentiated gastric cancer tissues, with a scattered distribution in the cytoplasm, suggesting a weak positive expression of NF-кB p50 (Figure 4).

\section{Correlation analysis between KPC1 expression and clinicopathological factors}

In 159 cases of gastric cancers and 56 cases of normal mucosa as control, KPC1 expression was significantly correlated with tumor differentiation, metastasis, and TNM staging $(\mathrm{P}<0.05)$, but not with age and lesion size $(\mathrm{P}>0.05)$ (Table 1).

\section{Correlation analysis of $\mathrm{NF}-\kappa \mathrm{B}$ p50 expression and clinicopathological factors}

In 159 cases of gastric cancer and 56 cases of normal mucosa as control, NF- $\mathrm{\kappa B}$ p50 expression was significantly correlated with tumor differentiation, metastasis, and TNM staging $(\mathrm{P}<0.05)$, but not with age or lesion size $(\mathrm{P}>0.05)$ (Table 2). 


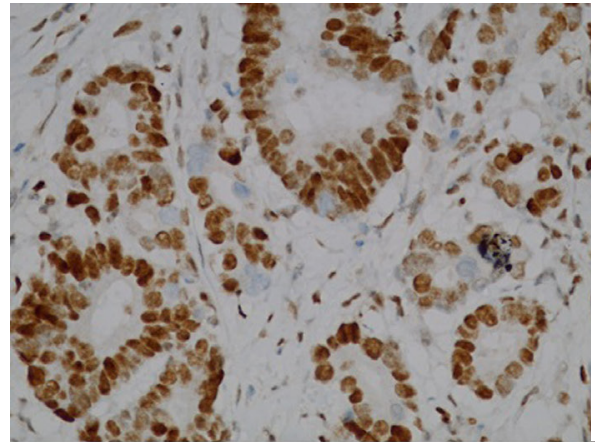

Highly differentiate (400x)

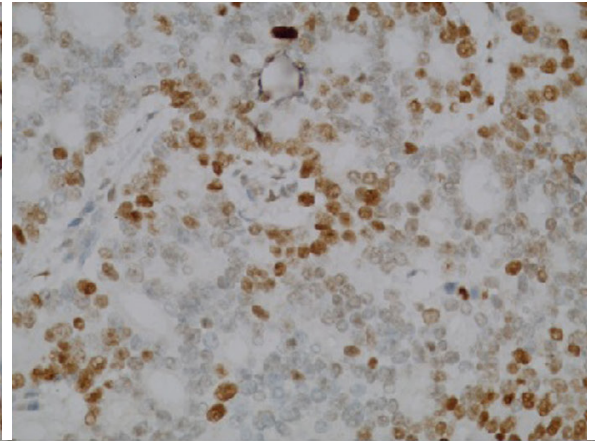

Moderately differentiated (400x)

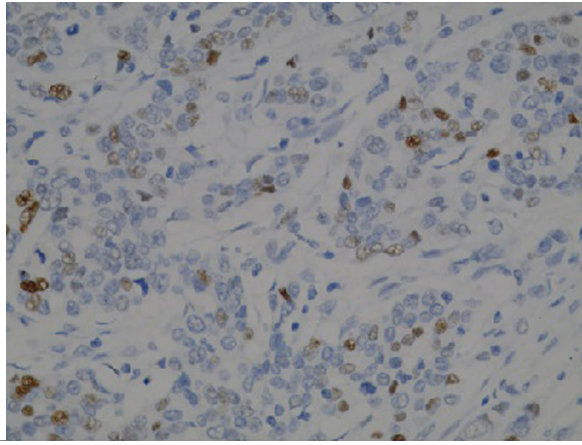

Poorly differentiated (400x)

Figure 3 Immunohistochemical detection of KPC1 expression in various differentiated gastric cancer tissues. KPC1, Kip1 ubiquitinationpromoting complex 1 .

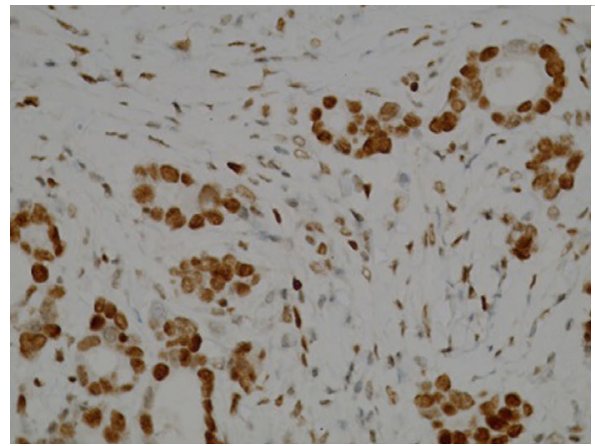

Highly differentiated (400x)

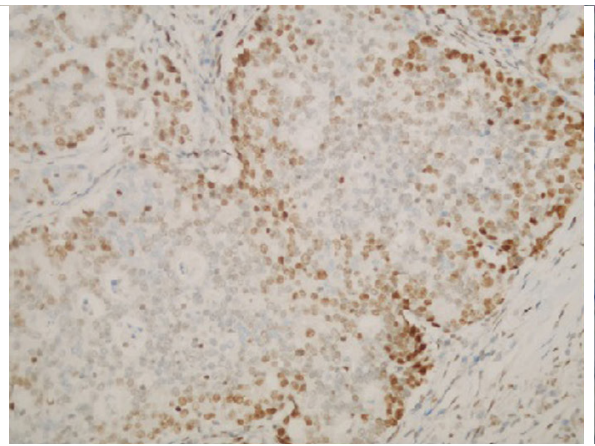

Moderately differentiated (400x)

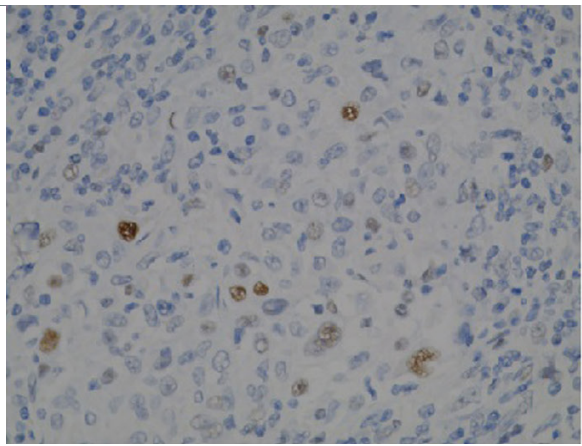

Poorly differentiated (400x)

Figure 4 Immunohistochemical detection of NF- $\mathrm{B}$ p50 expression in various differentiated gastric cancer tissues.

\section{Correlation between KPC1 and cytoplasmic NF- $\mathrm{kB}$ p50}

The correlation between KPC1 and cytoplasmic NF- $\kappa \mathrm{B}$ p50 was determined by Spearman's correlation analysis, which showed that KPC1 was positively correlated with the expression of NF- $\kappa \mathrm{B}$ p50 $(\mathrm{r}=0.427, \mathrm{P}<0.05)$. The scatter plot in Figure 5 shows a positive correlation. Immunohistochemistry detected $\mathrm{KPC} 1$ and $\mathrm{NF}-\kappa \mathrm{B}$ p 50 expression in the same gastric cancer tissue (Figure 6).

\section{KCP1 mRNA and NF-кB $p 50 m R N A$ expression analysis by RT-PCR}

KCP1 mRNA levels were significantly reduced in the 3 differentiated gastric cancer cell lines, and the expression of KCP1 was significantly different among the cell lines. The gray ratio of KCP1 to GAPDH showed a low expression in the poorly differentiated and highly tumorigenic MGC-803 cell lines, higher expression in the moderately differentiated
SGC-7901 cell line, and the highest expression in the highly differentiated and poorly tumorigenic AGS cell line (Figure 7).

$\mathrm{NF}-\kappa \mathrm{B}$ p50 mRNA levels were significantly reduced in the 3 differentiated gastric cancer cell lines. Meanwhile the expression of $\mathrm{NF}-\kappa \mathrm{B}$ p 50 was significantly different across these cell lines and was correlated with KCP1 expression. The gray ratio of NF- $\mathrm{kB}$ p50 to GAPDH showed low expression in the poorly differentiated and highly tumorigenic MGC-803 cell lines, higher expression in the moderately differentiated SGC-7901cell line, and the highest expression in the highly differentiated and poorly tumorigenic AGS cell line (Figure 8).

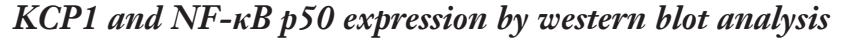

We identified a KCP1 band at the expected size of $160 \mathrm{kDa}$. The expression in the different cell lines was differed significantly. The gray ratio of protein to $\beta$-actin 
Table 1 Relationship between the expression of KCP1 and the clinicopathological parameters of gastric cancer

\begin{tabular}{|c|c|c|c|c|c|}
\hline Pathological parameters & $\mathrm{n}$ & \multicolumn{4}{|c|}{$\mathrm{KPC} 1$} \\
\hline \multicolumn{6}{|l|}{ Age (yrs) } \\
\hline$\leq 53$ & 47 & 35 & 12 & 0.001 & 1.000 \\
\hline$>53$ & 112 & 88 & 24 & & \\
\hline$I-I I$ & 69 & 40 & 29 & 5.112 & 0.023 \\
\hline III-IV & 90 & 72 & 8 & & \\
\hline \multicolumn{6}{|l|}{ Tumor differentiation } \\
\hline Control (normal mucosa) & 19 & 1 & 18 & 30.792 & 0.000 \\
\hline Poorly differentiated & 53 & 47 & 6 & & \\
\hline \multicolumn{6}{|l|}{ Metastasis } \\
\hline No & 102 & 38 & 64 & 16.912 & 0.000 \\
\hline Yes & 57 & 50 & 7 & & \\
\hline \multicolumn{6}{|l|}{ Diameter of lesions } \\
\hline$<1 \mathrm{~cm}$ & 109 & 22 & 87 & 0.201 & 0.713 \\
\hline$\geq 1 \mathrm{~cm}$ & 50 & 17 & 33 & & \\
\hline
\end{tabular}

KCP1, Kip1 ubiquitination-promoting complex 1; TNM, tumor-node-metastasis.

showed that the highly differentiated and poorly tumorigenic AGS cell line had the highest expression, followed by the moderately differentiated SGC-7901 cell line. The expression was very low in the poorly differentiated and highly tumorigenic MGC-803 cell lines (Figure 9).

We identified an NF- $\mathrm{NB}$ p50 band at the expected size of $50 \mathrm{kDa}$. The expression in the different cell lines was significantly different. The gray ratio of protein to $\beta$-actin showed that the highly differentiated and poorly tumorigenic AGS cell line had the highest expression, followed by the moderately differentiated SGC-7901 cell line. The expression was very low in the poorly differentiated and highly tumorigenic MGC-803 cell lines (Figure 9). These findings were consistent with the expression of KCP1.

\section{KCP1 and NF- $\mathrm{NB}$ p50 mediates gastric carcinoma cell proliferation}

Cell proliferation was measured by the WST-1 assay. We can infer that the expression of tumor suppressor genes is mediated by KCP1 and NF- $\mathrm{kB}$ p50, as KCP1 siRNA and SN50 inhibited KCP1 and NF- $\kappa \mathrm{B}$ p50 effects on MCG803 cells and increased their proliferation (Figure 10).

\section{Discussion}

The NF- $\kappa \mathrm{B}$ protein family is a class of transcription factors that plays a crucial part during the development of immunity, inflammation, and oncogenesis. The NF-кB/Rel family has 5 members: p65 (RelA), RelB, c-Rel,

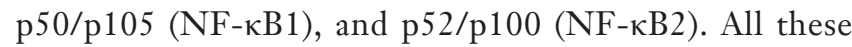
family members contain a highly conserved Rel homology region (Rel homology domain, RHD) containing 300 amino acids at the $\mathrm{N}$-terminus. The main function of the RHD domain is to form dimers, binding to IкBs and DNA at the same time. It also includes the nuclear localization signal (NLS), and activated NF- $\kappa B$ can enter the nucleus by mediating the NLS. p65 (RelA), RelB, and c-Rel include transcriptional activation transactivation 
Table 2 Relationship between the expression of NF-кB p50 and the clinicopathological parameters of gastric cancer

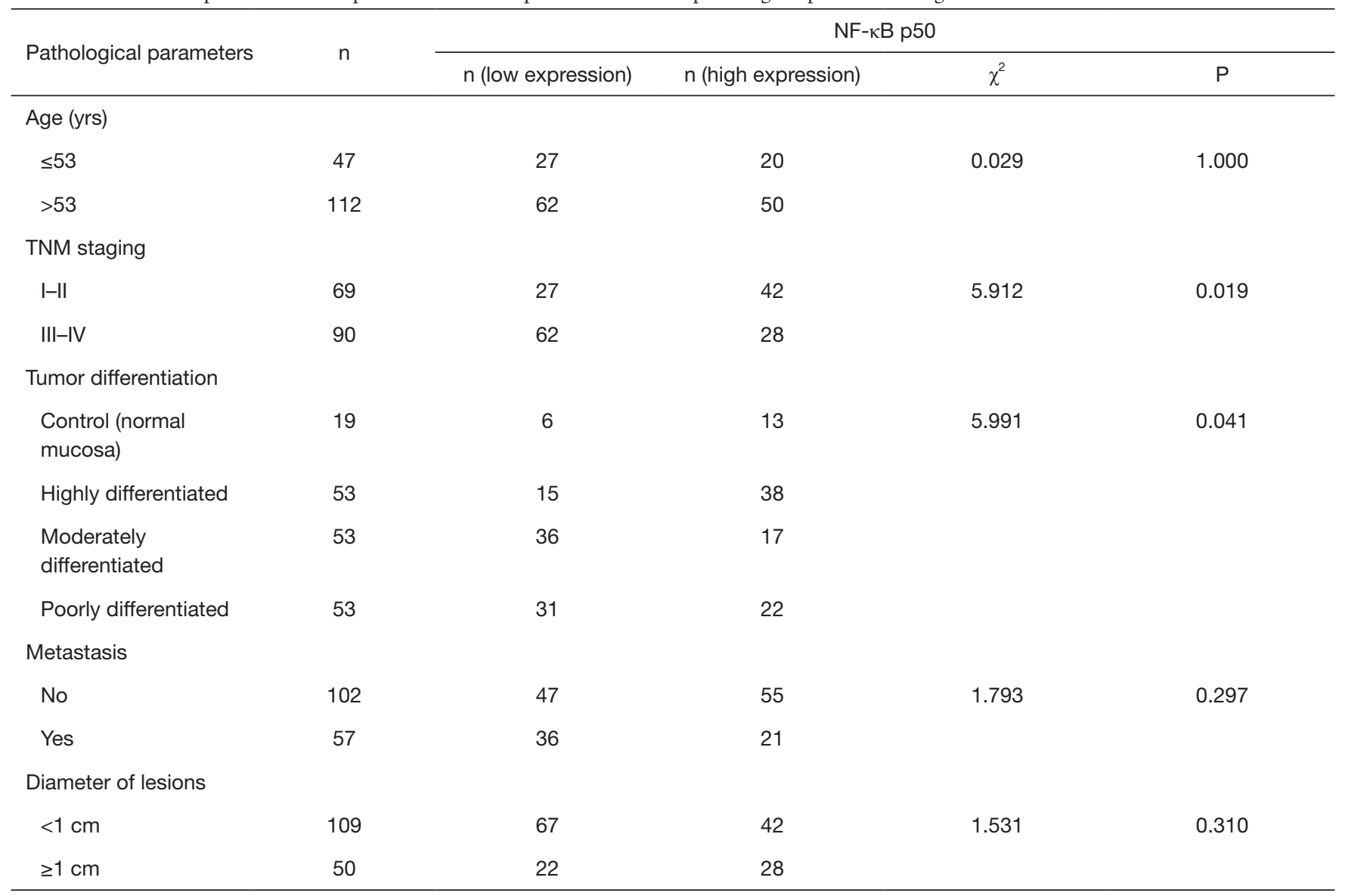

TNM, tumor-node-metastasis.

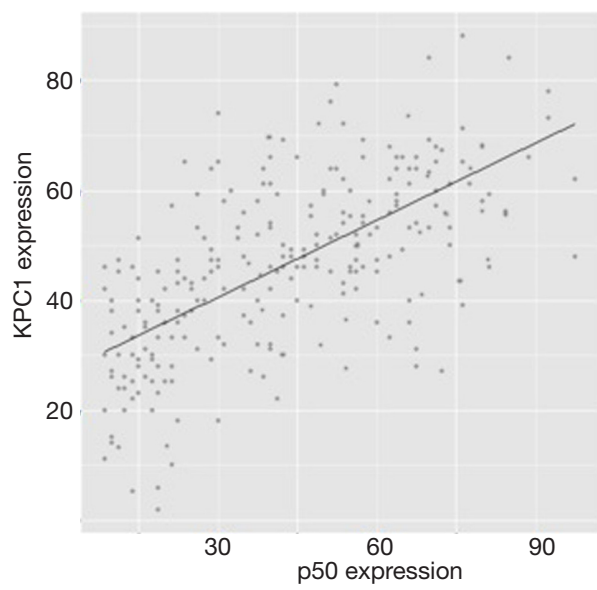

Figure 5 Correlation analysis between $\mathrm{KPC} 1$ and $\mathrm{NF}-\kappa \mathrm{B}$ p50. KPC1, Kip1 ubiquitination-promoting complex 1. domains (TADs) which can interact with many kinds of basic transcription factors. Their transcriptional function can only be activated under the action of the NF- $\kappa \mathrm{B}$ dimer by p65 (RelA), RelB, and c-Rel. However, p50 and p52, due to the absence of TAD, form a dimer that inhibits the transcription of target genes.

The UPP is one of the most important mechanisms of protein degradation due to its selectivity. It was found that this pathway-mediated regulation of cellular protein degradation is complex and sophisticated, and plays an important role in cell regulation. Through cell degradation, the activators and/or inhibitors of each pathway play a role in upregulation or downregulation.

There are generally 2 ways in which $\mathrm{NF}-\kappa \mathrm{B}$ family proteins are activated and then enter the nucleus from the 


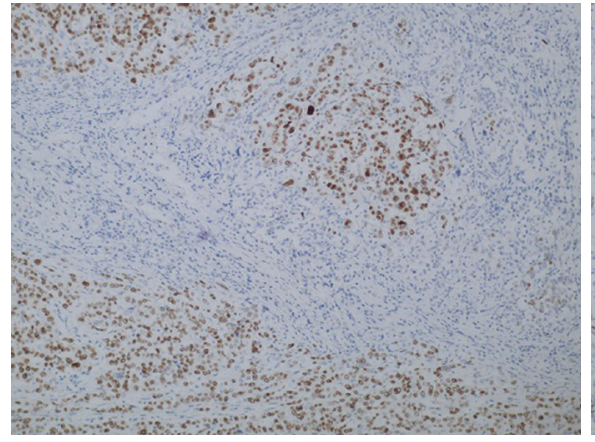

KPC1 expression (100x)

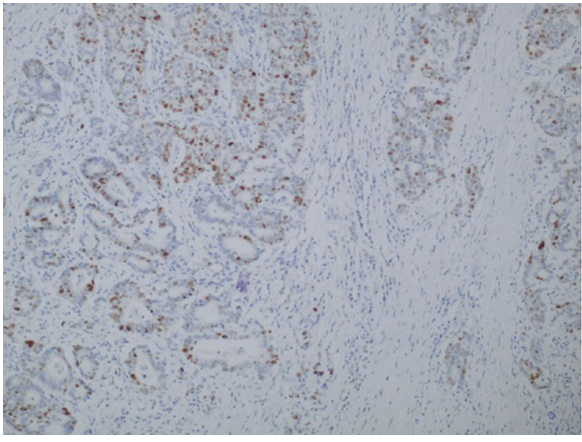

NF-kB p50 expression (100x)

Figure 6 Expression of KPC1 and NF-kB p50 in the same tissue specimen. KPC1, Kip1 ubiquitination-promoting complex 1.

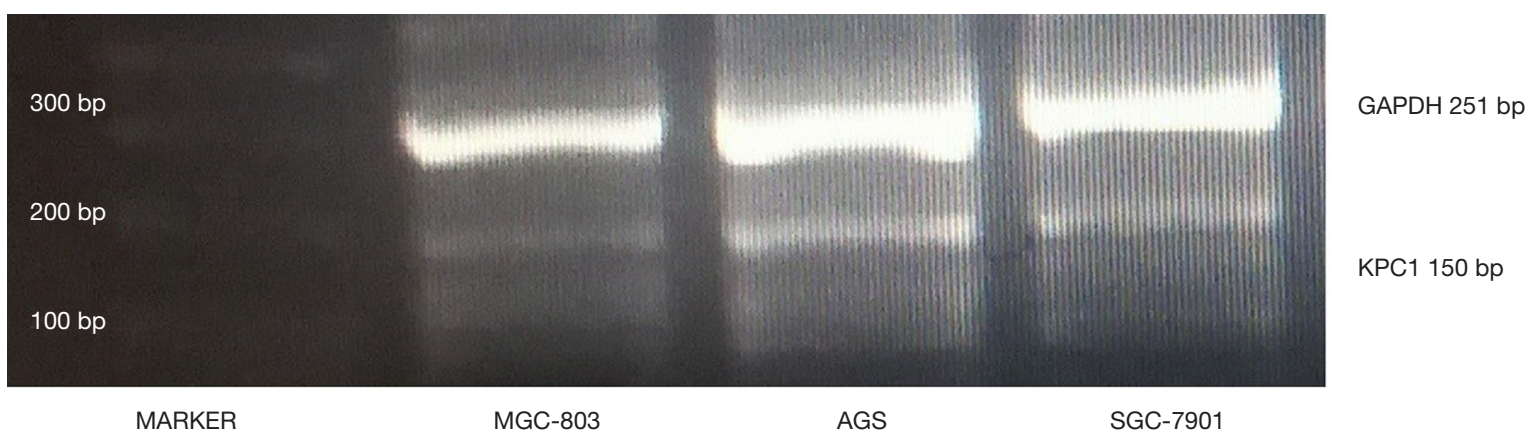

Figure 7 KCP1 mRNA expression in different gastric cancer cell lines. KPC1, Kip1 ubiquitination-promoting complex 1.

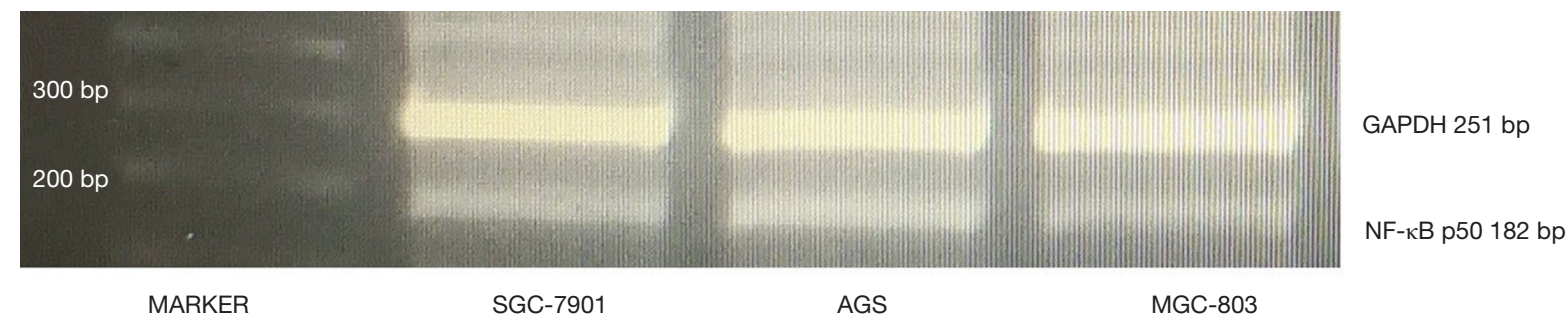

Figure $8 \mathrm{NF}-\mathrm{kB}$ p50 mRNA expression in different gastric cancer cell lines.

cytoplasm. Among the classical pathways, the heterodimers consisting of $\mathrm{p} 65 / \mathrm{p} 50$, which bind to $\operatorname{I\kappa B} \alpha$, cannot enter the nucleus. However, following a burst of external stimulation, signal is transducted to the IкB kinase complex, then serine at positions 32 and 36 of IкB $\alpha$ is phosphorylated. Phosphorylated IкB $\alpha$ is ubiquitinated and can be degraded by the $26 \mathrm{~S}$ proteasome. During this procedure, the UPP plays an important role and allows NF- $\mathrm{\kappa B}$ to enter the nucleus and play a transcriptional role in the nucleus via the exposure of the NLS of the NF- $\kappa$ B complex (13-18).

The present study found that KPC1 with ubiquitin ligase

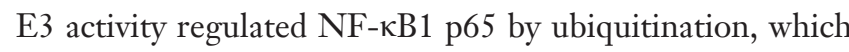
resulted in degradation through the UPP, and then formed active p50 following this degradation. p50 can promote tumor suppressor signal expression, and high expression can down-regulate p65 and oncogene expression.

Kravtsova-Ivantsiv et al. (10) demonstrated that KCP is composed of 2 subunits, KCP1 and KCP2. In their study, after cleavage of rabbit reticulocytes, fractionated the lysates and used the isotope-labeled p105 mutant in order to find the KPC family which caused p105 ubiquitination. In order to verify that KPC regulated the ubiquitination of 
p105, labeled p105 was used as a substrate, and experiments were performed using wild-type and mutant KPC1, which indicated that KPC1 did regulate ubiquitination of p105 through a specific regulation mode. Even p100, which is highly homologous to p105, cannot be ubiquitinated. In addition, co-immunoprecipitation assays showed that $\mathrm{KPC} 1$ can bind to and interact with p105. KPC1 is likely

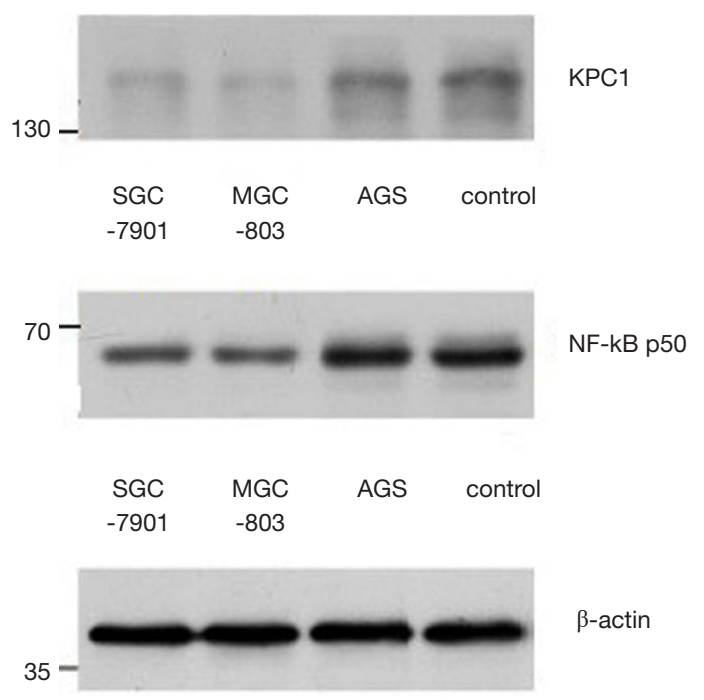

Figure $9 \mathrm{KCP} 1$ and $\mathrm{NF}-\kappa \mathrm{B}$ p50 protein expression in different cell lines (with $\beta$-actin as the internal reference). KPC1, Kip1 ubiquitination-promoting complex 1 . to play a role as an ubiquitin ligase during the regulation of p105 ubiquitination by specifically binding to p105. In cycloheximide experiments using siRNA, Kravtsova-Ivantsiv et al. found that KCP1 increased the production of $\mathrm{p} 50$ by promoting p105 to generate $\mathrm{p} 50$. In experiments using both wild-type and mutant KPC1, it was demonstrated that only wild-type KPC1 promoted p105 production to generate $\mathrm{p} 50$. In addition, co-immunoprecipitation assays showed that IKK $\beta$ did not change the binding of p105S927A to $\mathrm{KPC} 1$, indicating that the binding site of p105 to KPC1 was not related to IKK $\beta$. Furthermore, phosphorylated p105 was more susceptible to ubiquitination by KPC1. Therefore, KPC1 can interact with phosphorylated p105 to promote the production of $\mathrm{p} 50$, which is independent of IKK $\beta$. KPC2 is part of KPC1 in heterodimeric ligase complexes. Kravtsova-Ivantsiv et al. (10) showed that KPC2 had the effect of de-ubiquitination and could be recovered by KPC1 when using p105 as the substrate, regardless of the order of the addition of KPC1 and KCP2. This was the opposite to the effect of KPC1 on p105 ubiquitination and did not affect KPC1 expression.

The above experiments were carried out with animal and cell models, and were not based on human data. For digestive system tumors, there are no reliable data on whether KPC1 affects NF- $\mathrm{KB}$ transcription.

In the present study, we investigated the different pathological types of tissue sections from 159 gastric

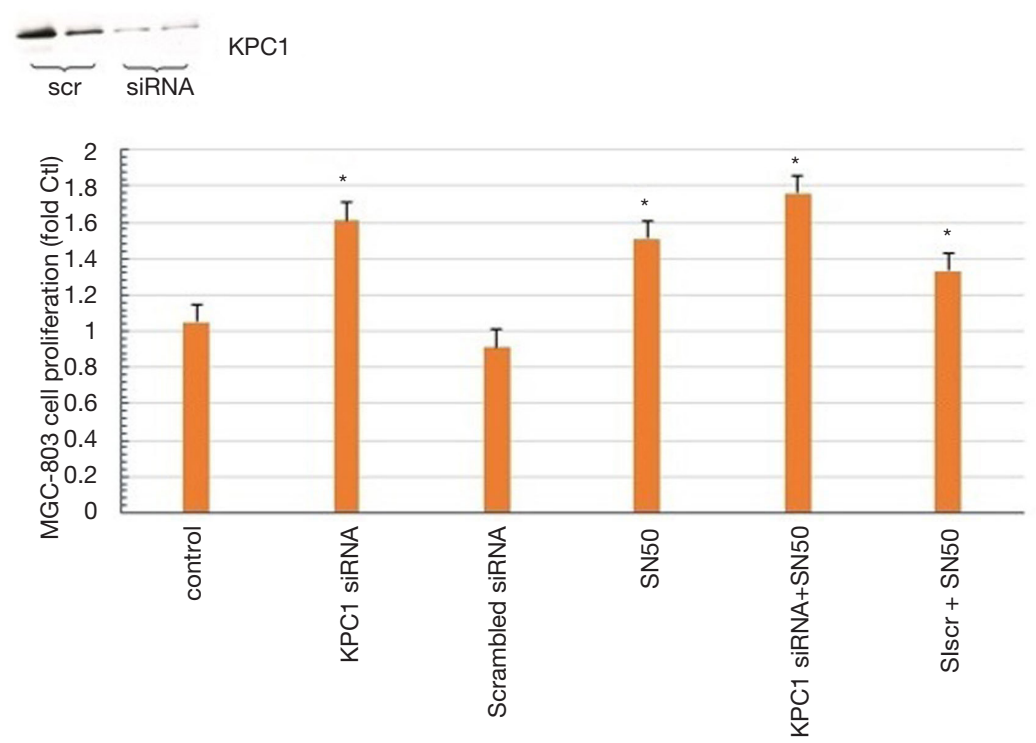

Figure $10 \mathrm{KCP} 1$ siRNA and SN50 inhibited the effects of KCP1 and NF- $\mathrm{KB}$ p50 MGC-803 on cell proliferation. KCP1 siRNA (20 nmol/L), or scrambled oligonucleotides for 48 hours (*, $\mathrm{P}<0.05$, compared with control cells). 
cancer patients, and the results showed that KPC1 was localized in the cytoplasm, and its expression was highest in patients with a high degree of tumor differentiation. Immunohistochemistry showed that KPC1 expression was significantly correlated with gastric cancer TNM staging, along with tumor differentiation and metastasis, but not with patient age and lesion size. KPC1 expression was also significantly correlated with the prognosis of patients with gastric cancer, as the survival time was significantly longer in patients with high KCP1 expression than in those with lower KCP1 expression, which had some significance in the evaluation of postoperative prognosis.

However, NF- $\mathrm{kB}$ p50 was found to be scattered in tissue sections and localized in the cytoplasm and/or nucleus, with higher expression in the cytoplasm and lower expression in the nucleus in patients with highly differentiated tumors. In poorly differentiated tissues, $\mathrm{NF}-\kappa \mathrm{B}$ p50 was distributed in the nucleus and was weakly expressed, and was also expressed in the cytoplasm. These findings demonstrate that the NF- $\mathrm{BB}$ complex in poorly differentiated tumors played a regulatory role when present in the nucleus. Thus, its role in tumor regulation may be controlled if the presence of the NF- $\mathrm{kB}$ complex in the nucleus is reduced, which may inhibit tumor development. It was found that NF- $\kappa$ B p50 expression was correlated with TNM staging and tumor differentiation, but not with age, tumor size, and metastasis, which was similar to correlates of KPC1 expression following pathological analysis; however, the results relating to the tumor metastasis situation were not similar.

In addition, Spearman's correlation analysis showed that there was a positive correlation between KPC1 and the expression of $\mathrm{NF}-\kappa \mathrm{B}$ p50 in the cytoplasm. The above results suggest that KPC1 is co-expressed with NF-кB p50 in the cytoplasm. Taken together, these findings indicate that KPC1 and NF- $\mathrm{\kappa B}$ p50 may be tumor suppressors in the mechanism of gastric carcinogenesis, and may interact with each other. Our study results were in accordance with recent reports on other tumor cell systems.

Kravtsova-Ivantsiv et al. (10) found that KPC1 overexpression significantly inhibited the rate of colony formation in breast cancer MB-MDA 231, human osteosarcoma U2OS, and human brain astrocytoma U87-MG cells, whereas mutant KPC1 (no ubiquitin ligase activity) did not have an inhibitory effect. These findings show that KPC1 can inhibit the growth of tumor cells through its ubiquitin ligase activity. Moreover, KPC1 plays a role in the conversion of p105 to p50, which may promote tumor suppressor gene expression and inhibit tumor growth. Also, the low expression of KPC1 was associated with decreased p50 and an increased incidence of gastric cancer. Current research in this area is focused primarily on the function of the signaling pathway which activates upstream of NF- $\kappa \mathrm{B}$ or its other subunits, such as RelA (p65), and these data may suggest a key role for NF- $\mathrm{KB} 1$ in inflammation and offer a new strategy for targeted therapy of cancer signaling pathways.

In the present study, we found through RT-PCR and western blotting that the expression of KCP1 in gastric cancer cell lines was low.

We selected 3 gastric cancer cell lines for the experiments: SGC-7901, MGC-803, and AGS. Of these, AGS is a well-differentiated gastric adenocarcinoma cell line, SGC-7901 is a moderately differentiated cell line, and MGC-803 is a poorly differentiated cell line. According to their tumorigenicity in nude mice, they were classified as strong tumorigenic cell lines (SGC-7901, MGC-803) and weak tumorigenic cell lines (AGS). By choosing cell lines with different levels of differentiation and tumorigenicity, we could ensure that the experimental data were more convincing.

The expression levels of KPC1 and NF- $\mathrm{kB}$ p50 mRNA and protein were different. Expression was low in the poorly differentiated and highly tumorigenic MGC-803 cells. Expression in the moderately differentiated SGC-7901 cells was higher, and expression in the highly differentiated and less tumorigenic AGS cells was highest. This was consistent with the clinical data by immunohistochemistry, and the effect of KPC1 and NF- $\mathrm{KB}$ p50 on gastric cancer in vitro showed that there was a correlation between them.

In this study, we investigated the relationship between the expression of cytoplasmic NF-kB p50 and KPC1 which can degrade NF- $\kappa \mathrm{B}$ p50. The results reveal that they were co-expressed in gastric cancer tissues with a high degree of differentiation, which resulted in a better prognosis and inhibited tumor growth. However, the underlying mechanisms require further investigation. Further studies on the effect of KPC1 on p50 target gene expression will be carried out in the future.

KPC1 and cytoplasmic NF- $\mathrm{kB}$ p50 are synergistically expressed in gastric cancer and promote the expression of tumor suppressor genes, thereby limiting the growth of tumor cells and possibly inhibiting the malignant development of gastric cancer. These insights may provide new research directions and potential therapeutic targets for tumor treatment. 


\section{Acknowledgments}

Our sincere appreciation to all participants and staff of the Laboratory of Digestion at Xin Hua Hospital for their assistance and cooperation throughout this study.

Funding: This study was additionally supported by the National Natural Science Foundation of China (grant number 81472844).

\section{Footnote}

Conflicts of Interest: The authors have no conflicts of interest to declare.

Ethical Statement: The authors are accountable for all aspects of the work in ensuring that questions related to the accuracy or integrity of any part of the work are appropriately investigated and resolved. The study was approved by the ethics committee of Xin Hua Hospital affiliated to Shanghai Jiao Tong University School of Medicine, and written informed consent of each patient enrolled during the research was acquired.

Open Access Statement: This is an Open Access article distributed in accordance with the Creative Commons Attribution-NonCommercial-NoDerivs 4.0 International License (CC BY-NC-ND 4.0), which permits the noncommercial replication and distribution of the article with the strict proviso that no changes or edits are made and the original work is properly cited (including links to both the formal publication through the relevant DOI and the license). See: https://creativecommons.org/licenses/by-nc-nd/4.0/.

\section{References}

1. Bray F, Ren JS, Masuyer E, Ferlay J. Global estimates of cancer prevalence for 27 sites in the adult population in 2008. Int J Cancer 2013;132:1133-45.

2. Ferlay J, Shin HR, Bray F, Forman D, Mathers C, Parkin DM. Estimates of worldwide burden of cancer in 2008: GLOBOCAN 2008. Int J Cancer 2010;127:2893-917.

3. Jemal A, Center MM, DeSantis C, Ward EM. Global patterns of cancer incidence and mortality rates and trends. Cancer Epidemiol Biomarkers Prev 2010;19:1893-907.

4. Soerjomataram I, Lortet-Tieulent J, Parkin DM, et al. Global burden of cancer in 2008: a systematic analysis of disability-adjusted life-years in 12 world regions. Lancet 2012;380:1840-50.

5. Ohta M, Inoue H, Cotticelli MG, et al. The FHIT gene, spanning the chromosome 3 p14.2 fragile site and renal carcinoma-associated $\mathrm{t}(3 ; 8)$ breakpoint, is abnormal in digestive tract cancers. Cell 1996;84:587-97.

6. Zhang H, Song Y, Yang C, et al. UHRF1 mediates cell migration and invasion of gastric cancer. Biosci Rep. 2018;38:1-8.

7. Li Z, Nabel GJ. A new member of the I kappaB protein family, I kappaB epsilon, inhibits RelA (p65)-mediated NF-kappaB transcription. Mol Cell Biol 1997;17:6184-90.

8. Kamura T, Hara T, Matsumoto M, et al. Cytoplasmic ubiquitin ligase KPC regulates proteolysis of p27(Kip1) at G1 phase. Nat Cell Biol 2004;6:1229-35.

9. Han Y, Wu A, Chen Y, et al. The regulation effect of RNF123 on the stability of p27 (Kip1) protein in ovarian cancer cells. Journal of Nanjing Medical University 2015;35:666-9.

10. Kravtsova-Ivantsiv Y, Shomer I, Cohen-Kaplan V, et al. KPC1-mediated ubiquitination and proteasomal processing of NF-kB1 p105 to p50 restricts tumor growth. Cell 2015;161:333-47.

11. Perkins ND. Post-translational modifications regulating the activity and function of the nuclear factor kappa B pathway. Oncogene 2006;25:6717-30.

12. Zhu BS, Xing CG, Lin F, et al. Blocking NF- $\kappa B$ nuclear translocation leads to p53-related autophagy activation and cell apoptosis. World J Gastroenterol 2011;17:478-87.

13. Baeuerle PA, Baltimore D. A 65-kappaD subunit of active NF-kappaB is required for inhibition of NF-kappaB by I kappaB. Genes Dev 1989;3:1689-98.

14. Perkins ND. The diverse and complex roles of NF- $\mathrm{kB}$ subunits in cancer. Nat Rev Cancer 2012;12:121-32.

15. Kuwata H, Matsumoto M, Atarashi K, et al. IkappaBNS inhibits induction of a subset of Toll-like receptor-dependent genes and limits inflammation. Immunity 2006;24:41-51.

16. Trinh DV, Zhu N, Farhang G, et al. The nuclear I kappaB protein I kappaB zeta specifically binds NF-kappaB p50 homodimers and forms a ternary complex on kappaB DNA. J Mol Biol 2008;379:122-35.

17. Ghosh S, May MJ, Kopp EB. NF-kappa B and Rel proteins: evolutionarily conserved mediators of immune responses. Annu Rev Immunol 1998;16:225-60.

18. Van Antwerp DJ, Martin SJ, Kafri T, et al. Suppression of TNF-alpha-induced apoptosis by NF-kappaB. Science 1996;274:787-9.

Cite this article as: Zhou M, Qu CY, Ding WJ, Cai XL, Shen YJ, Chen YW, Xu LM. Preliminary study on the inhibitory effect of tumor suppressor gene KPC1 on the proliferation in gastric carcinoma cell. Ann Transl Med 2020;8(6):343. doi: 10.21037/atm.2020.02.79 